\title{
New Results on Turbulence Modeling for Free-Space Optical Systems
}

\author{
Nestor D. Chatzidiamantis*, Harilaos G. Sandalidis ${ }^{\dagger}$, George K. Karagiannidis*, Stavros A. Kotsopoulos ${ }^{\ddagger}$ \\ and Michail Matthaiou ${ }^{\S}$ \\ *Department of Electrical and Computer Engineering, Aristotle University of Thessaloniki, Thessaloniki, Greece, emails: \{nestoras, geokarag\}@ auth.gr

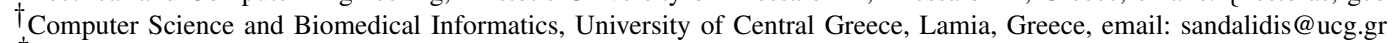 \\ ${ }_{\ddagger}$ Department of Electrical and Computer Engineering, University of Patras, Patras, Greece, email: kotsop@ee.upatras.gr

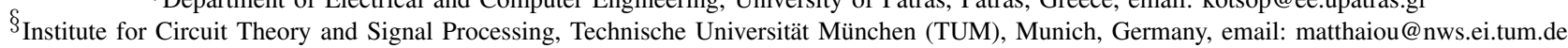

\begin{abstract}
In this paper, we propose a statistical channel model, named as Double-Weibull, to describe the irradiance fluctuations in moderate and strong turbulence for free-space optical (FSO) systems. The proposed stochastic model is based on the scintillation theory and derived via the product of two Weibull random variables. Closed-form expressions of probability and cumulative density functions are provided in terms of Meijer's G-function. We also compare the new model with the classical gamma-gamma model and assess their accuracy via a set of simulations when both plane and spherical waves are considered. We finally evaluate the performance of an FSO system over the Double-Weibull turbulence channel and derive closed-form expressions for the bit-error rate, assuming intensitymodulation/direct detection with On-Off keying, and the outage probability.
\end{abstract}

\section{INTRODUCTION}

Free-Space optical (FSO) communication systems have recently attracted considerable attention for a variety of applications, since they offer a license-free and cost-effective access performance. However, FSO systems are subject to atmospheric turbulence, which originate from variations in the refractive index of the transmission medium due to inhomogeneities in temperature and pressure changes. As a consequence, rapid fluctuations at the received optical signal occur, known as fading or scintillation, which impair and degrade system performance particularly for link ranges of $1 \mathrm{~km}$ and above [1]. Scintillation is characterized as weak when the irradiance variance is less than unity and strong when greater. The range of the strong scintillation regime for which irradiance variance decreases when turbulence level increases, is called saturation of scintillation [2].

The identification of a tractable probability density function (pdf) to describe atmospheric turbulence is important in order to study the performance characteristics of an FSO system. Over the last decades, a number of statistical models have been proposed for different degrees of turbulence severity. Under weak irradiance fluctuations and long propagation distances, the most widely used model is the log-normal one, which provides a good fit with experimental measurement data. Discrepancies, however, occur as the turbulence strength increases. In this case, irradiance statistics can be more effectively modeled using the negative exponential distribution, or the K-channel model [1].
In [3], Al-Habash et al. proposed the gamma-gamma distribution as an efficient model for atmospheric turbulence channels. This model is based on the scintillation and modified Rytov theories, introduced in [4], and considers irradiance fluctuations as the product of small-scale and large-scale fluctuations, where both can be statistically defined via the gamma distribution. The gamma-gamma channel model is a two parameter distribution which depends directly on the atmospheric parameters, and has the ability to describe turbulence-induced fading under every degree of turbulence severity.

In this paper, we introduce a new efficient turbulence model, named as Double-Weibull, for describing moderate and strong irradiance fluctuations. The parameters of the proposed model pdf are tied directly to the atmospheric parameters. Based on scintillation and modified Rytov theories, we consider the composite fluctuations as the product of small-scale and large-scale fading, both statistically defined by the Weibull distribution, and derive closed-form expressions for their pdf and cumulative density (cdf) functions in terms of Meijer's G-function. In addition, we perform a detailed comparison of our model with the above mentioned gamma-gamma model. This is accomplished by using some plane and spherical wave pdf simulation data over moderate and strong turbulence conditions that include inner scale effects. This set of measured data can be found in [5] and [6], respectively, while it was also used in the seminal paper of Al Habash et al. [3], for the derivation of the gamma-gamma distribution. Our results clearly demonstrate that the Double-Weibull model fits the experimental data with better accuracy, under all the considered scenarios. Finally, we study the performance of a typical FSO system, which uses intensity modulation/direct detection (IM/DD) with On-Off Keying (OOK), after introducing the new channel model. Analytical closed-form expressions for the average bit-error rate (BER) and the outage probability are also provided.

The remainder of the paper is organized as follows. In Section II, the new Double-Weibull model is introduced as the product of small-scale and large-scale fluctuations. Closedform expressions for the pdf and cdf are also provided. In Section III, we compare the new pdf model with the gammagamma model and also with a set of simulation data assuming both plane and spherical waves. In Section IV, the performance of the FSO system with IM/DD with OOK over the new model 
is studied. Section V concludes the paper and summarizes the key findings.

\section{Double-Weibull STATISTICAL MODEL}

Let us consider a Weibull distributed random variable, $x$,with pdf given by [7, Eq. (17.1)]

$$
f_{x}(x)=\frac{\beta x^{\beta-1}}{\Omega} \exp \left(-\frac{x^{\beta}}{\Omega}\right),
$$

where $\beta>0$ is the distribution parameter related to the severity of the irradiance fluctuations and $\Omega>0$ is related to their average power. The $n$-order moment of the random variable $x$ is given by [7, Eq. (17.6)] as

$$
E\left\langle x^{n}\right\rangle=(\Omega)^{\frac{n}{\beta}} \Gamma\left(1+\frac{n}{\beta}\right),
$$

with $E\langle\cdot\rangle$ denoting expectation while $\Gamma(\cdot)$ is the well-known gamma function [8, Eq. (8.310.1)].

According to the scintillation theory [3], [4], the irradiance of the received optical wave can be modeled as the product of two types of fluctuations, i.e. $I=x y$, each arising from large and small scale turbulent eddies, respectively. It is assumed that $x, y$ are statistically independent random processes and, therefore, the second moment of the irradiance is expressed as

$$
E\left\langle I^{2}\right\rangle=\left\langle x^{2}\right\rangle\left\langle y^{2}\right\rangle=\left(1+\sigma_{x}^{2}\right)\left(1+\sigma_{y}^{2}\right),
$$

where $\sigma_{x}^{2}$ and $\sigma_{y}^{2}$ are normalized variances of $x$ and $y$, respectively. Without loss of generality, we assume $E\langle I\rangle=1$ or equivalently $E\langle x\rangle=1$ and $E\langle y\rangle=1$. Then the scintillation index is given by

$$
\sigma_{I}^{2}=\frac{E\left\langle I^{2}\right\rangle}{E\langle I\rangle^{2}}-1=\left(1+\sigma_{x}^{2}\right)\left(1+\sigma_{y}^{2}\right)-1 .
$$

Now, let us assume that each type of scale of the irradiance fluctuations is governed by the Weibull distribution, i.e. $x$, $y$ have the pdf of (1) with parameters $\beta_{1}, \Omega_{1}$ and $\beta_{2}, \Omega_{2}$, respectively. Then, using the generalized statistical model proposed in [9] and after making some basic simplifications on its parameters, the pdf of $I$ can be written as

$$
\begin{aligned}
& f_{I}(I)=\frac{\beta_{2} k(k l)^{\frac{1}{2}}}{(2 \pi)^{\frac{l+k}{2}-1}} I^{-1} \\
& \times G_{k+l, 0}^{0, k+l}\left[\left(\frac{\Omega_{2}}{I^{\beta_{2}}}\right)^{k} k^{k} l^{l} \Omega_{1}^{l} \mid \Delta(l ; 0), \Delta(k ; 0)\right],
\end{aligned}
$$

where $G_{p, q}^{m, n}[\cdot]$ is the Meijer's G-function defined in [8, eq. (9.301)], $\Delta(j ; x) \triangleq \frac{x}{j}, \ldots, \frac{x+j-1}{j}$ and $l$ and $k$ are positive integer numbers that satisfy

$$
\frac{l}{k}=\frac{\beta_{2}}{\beta_{1}} .
$$

Using [9, Eq. (10)] and after some simplifications, the cdf of $I$ can be obtained in closed form as follows

$$
\begin{aligned}
F_{I}(I) & =\frac{\sqrt{l k}}{(2 \pi)^{\frac{l+k}{2}-1}} \\
& \times G_{1, k+l+1}^{k+l, 1}\left[\frac{I^{\beta_{1} l}}{\left(\Omega_{1} l\right)^{l}\left(\Omega_{2} k\right)^{k}} \mid \Delta(l ; 1), \Delta(k ; 1), 0\right] .
\end{aligned}
$$

\section{COMPARISON With Simulation Data}

In this section, we investigate the suitability of the DoubleWeibull channel model to fit simulation data of plane and spherical waves, derived from [5] and [6], respectively, which impigne on a random medium characterized by homogeneous and isotropic Kolmogorov turbulence. We recall that the same data set was used by Andrews in [3], in order to introduce the gamma-gamma turbulence induced fading model.

The parameters of the Double-Weibull model in each case examined are defined using the first and second order moments of small and large scale irradiance fluctuations. The latter are directly tied to the simulated atmospheric conditions and depend on whether the simulation is based on plane or spherical waves. Hence, by relating the first and second order moments obtained from (2) with $\sigma_{x}^{2}$ and $\sigma_{y}^{2}$, the following expressions are derived, which determine the distribution parameters according to the atmospheric conditions

$$
\sigma_{i}^{2}=\frac{\Gamma\left(1+\frac{2}{\lambda_{i}}\right)}{\Gamma\left(1+\frac{2}{\lambda_{i}}\right)^{2}}-1
$$

and

$$
\Omega_{i}=\left(\frac{1}{\Gamma\left(1+\frac{1}{\beta_{i}}\right)}\right)^{\beta_{i}},
$$

where $i=1,2$ and $\sigma_{1}^{2}=\sigma_{x}^{2}, \sigma_{2}^{2}=\sigma_{y}^{2}$. Please note that (8) can be solved using [7, Eq. (17.51)], leading to

$$
\lambda_{i}=\sigma_{i}^{-1.0852} \text {. }
$$

\section{A. Plane Wave Data}

Figures 1-2 depict the simulation data provided by Flatté et al. in [5]. In their paper, the authors carried out exhaustive numerical simulations assuming plane wave propagation through homogeneous and isotropic Kolmogorov turbulence. The turbulence severity extends from moderate irradiance fluctuations, characterized by $\sigma_{\text {Rytov }}^{2}=2$, to the saturation regime, characterized by $\sigma_{\text {Rytov }}^{2}=25$. Based on their simulation results, Flatté et al. predicted some experimental pdfs of irradiance and depicted them in a way that reveals their salient features. Particularly, the logarithm of irradiance was chosen to illustrate the high and low irradiance tails [10]. Thus, sensitivity to the small irradiance fades is increased, while sensitivity to large irradiance peaks is decreased. Then, the experimental pdf plots were scaled by subtracting the mean value to center all distributions around the origin and by dividing by the square root of variance. The $y$-axis of 
the plots depicts the log-irradiance pdf multiplied by the square root of variance [5]. The simulation results showed that the experimental pdfs in the strong fluctuation regime lie between the log-normal and exponential distributions, and their moments lie between those of a log-normal exponential distribution and those of a $\mathrm{K}$ distribution [5].

On the same grounds, we have plotted the Double-Weibull pdf of the $\log$-irradiance as a function of $(\ln I-\langle\ln I\rangle) / \sigma$. The scaling parameters $\langle\ln I\rangle$ and $\sigma$ are the mean and the rms values of $\ln I$ calculated by averaging $\ln I$ over the gamma-gamma or Double-Weibull model. The parameters of the Double-Weibull model are calculated using (8) and (9), and the values of the variances of small and large scale fluctuations, $\sigma_{y}^{2}$ and $\sigma_{x}^{2}$, respectively, for plane waves. These variances are given by [3, Eq. (18)-(20)] depending on inner scale values. The gamma-gamma distribution is also plotted with its parameters being associated with the same atmospheric conditions.

In Fig. 1, we demonstrate the Double-Weibull model assuming moderate irradiance fluctuations, which are characterized by $\sigma_{\text {Rytov }}^{2}=2$ and $\frac{l_{o}}{R_{F}}=0.5$. Under these atmospheric conditions, its parameters are defined as $\beta_{1}=1.522, \beta_{2}=1.318$, $\Omega_{1}=1.171$ and $\Omega_{2}=1.114$. Moreover, $l$ and $k$ are selected as $l=6$ and $k=7$. Assuming the same atmospheric conditions, the parameters of the gamma-gamma model are $a=2.23$ and $b=1.706$. The comparison between the two models clearly demonstrates that the Double-Weibull model provides a better fit with the simulation data, especially at low probabilities of the logarithmic irradiance.

To further investigate the validity of the proposed model, one more case has been examined in Fig. 2. This figure depicts strong irradiance fluctuations, which are characterized by $\sigma_{\text {Rytov }}^{2}=25$ and $l_{o} / R_{F}=1$. The parameters of the DoubleWeibull model are defined as $\beta_{1}=1.2529, \beta_{2}=1.014$, $\Omega_{1}=1.0938, \Omega_{2}=1.006$ and $l$ and $k$ are selected as $l=4$ and $k=5$, whereas for the gamma-gamma distribution as $a=1.55$ and $b=1.03$. Once more, the Double-Weibull model matches the simulation data with satisfactory accuracy.

\section{B. Spherical Wave Data}

Figures 3-4 illustrate the simulation data provided by Hill and Frehlich in [6]. This set of numerical data was generated from simulation of spherical waves propagating through turbulent media. The atmospheric conditions spab a range from moderate irradiance fluctuations, characterized by $\sigma_{\text {Rytov }}^{2}=2$, to strong ones, characterized by $\sigma_{\text {Rytov }}^{2}=5$. In the same graphs, the gamma-gamma and Double-Weibull pdfs of the logarithm of irradiance are overlaid. These pdfs are plotted as a function of $\left(\ln I+0.5 \cdot \sigma^{2}\right) / \sigma$, where $\sigma$ is the square root of the variance of $\ln I$ and is directly calculated by averaging $\ln I$ over the Double-Weibull pdf model. In both figures, the $y$-axis depicts the pdf of the log-irradiance multiplied by $\sigma$.

The gamma-gamma and Double-Weibull model parameters have been evaluated using the variances of small and large scale fluctuations, $\sigma_{y}^{2}$ and $\sigma_{x}^{2}$ respectively, for spherical waves.

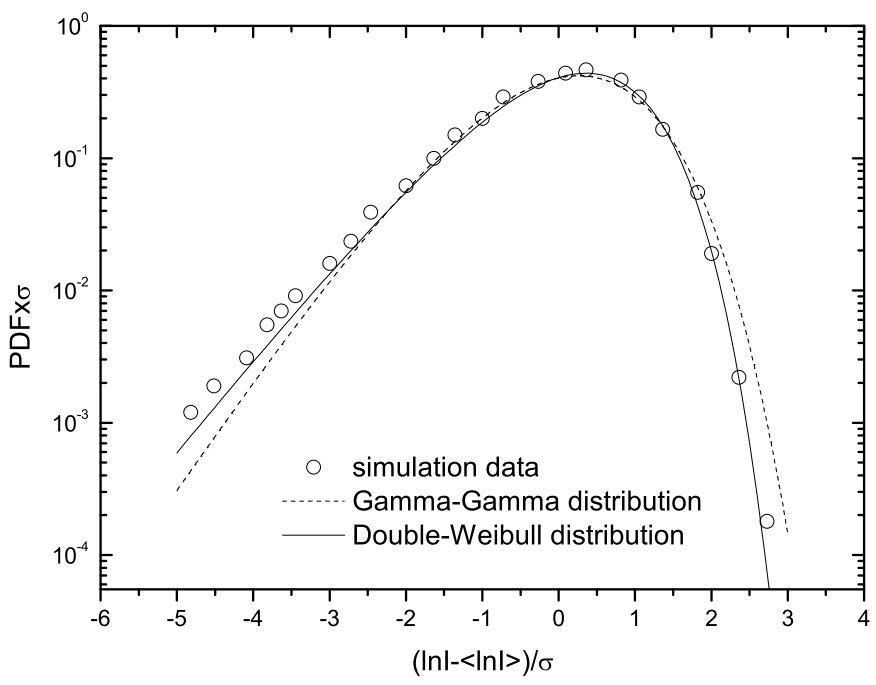

Fig. 1. Pdfs of the scaled log-irradiance for a plane wave assuming moderate irradiance fluctuations: $\sigma_{\text {Rytov }}^{2}=2$ and $l_{o} / R_{F}=0.5$.

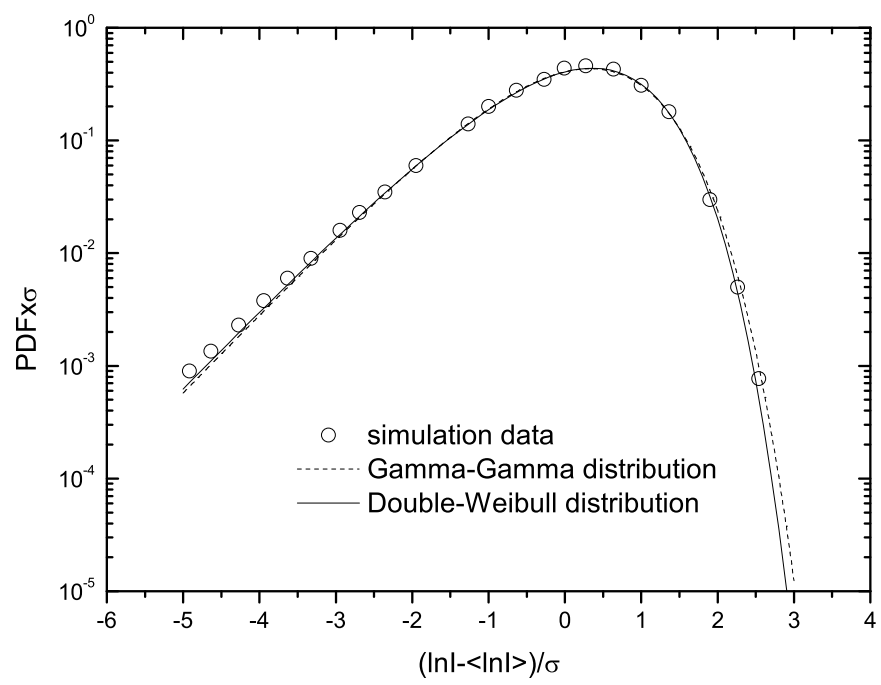

Fig. 2. Pdfs of the scaled log-irradiance for a plane wave assuming strong irradiance fluctuations: $\sigma_{\text {Rytov }}^{2}=25$ and $l_{o} / R_{F}=1$.

These variances were evaluated using [3, Eq. (23)-(27)] depending on inner scale values.

In Fig. 3, the Double-Weibull model is illustrated for moderate irradiance fluctuations characterized by $\sigma_{\text {Rytov }}^{2}=2$ and $l_{o} / R_{F}=0$. The model's parameters are defined as $\beta_{1}=1.511, \beta_{2}=1.315, \Omega_{1}=1.168, \Omega_{2}=1.113, l=7$ and $k=8$, while for the gamma-gamma one as $a=2.20$ and $b=1.70$. It is clearly observed that the Double-Weibull model provides a better fit with simulation data, especially for small irradiance values.

Likewise, the performance of the Double-Weibull model for strong irradiance fluctuations, characterized by $\sigma_{\text {Rytov }}=5$ and $l_{o} / R_{F}=1$, is examined in Fig. 4. Under these atmospheric conditions $\beta_{1}=0.695, \beta_{2}=1.092, \Omega_{1}=0.844$ and $\Omega_{2}=$ 1.037. Moreover, $l$ and $k$ are selected as $l=11$ and $k=7$. For 


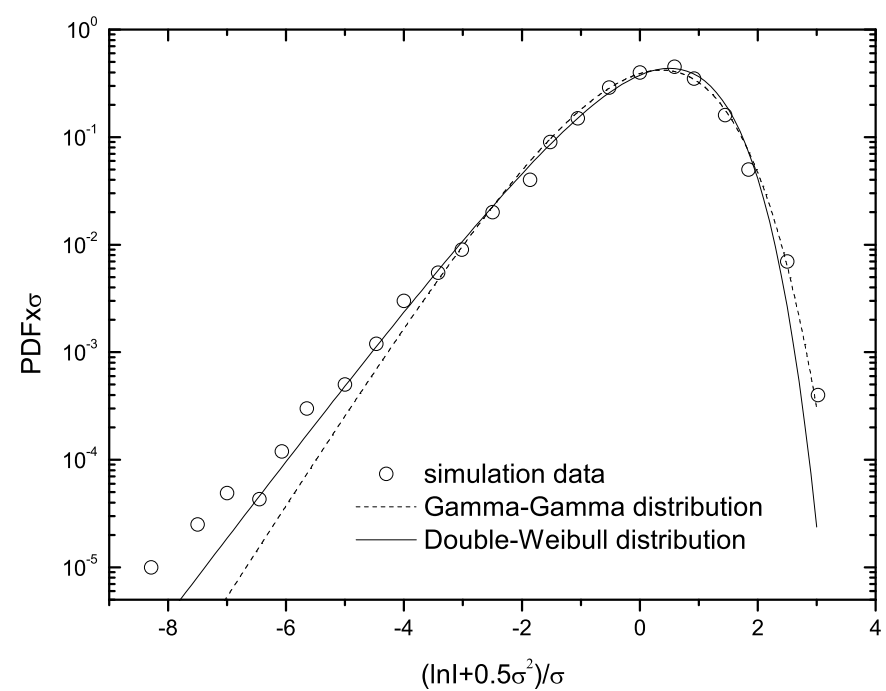

Fig. 3. Pdfs of the scaled log-irradiance for a spherical wave assuming moderate irradiance fluctuations: $\sigma_{\text {Rytov }}^{2}=2$ and $l_{o} / R_{F}=0$.

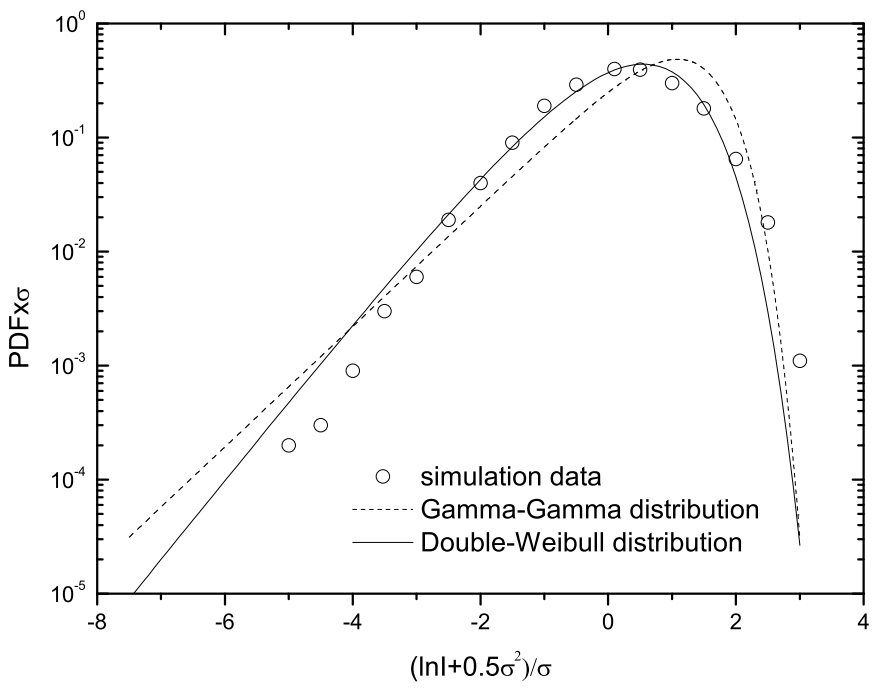

Fig. 4. Pdfs of the scaled log-irradiance for a spherical wave assuming strong irradiance fluctuations: $\sigma_{\text {Rytov }}^{2}=5$ and $l_{o} / R_{F}=1$.

the same atmospheric conditions, the parameters of gammagamma model are defined as $a=0.46$ and $b=1.19$. Once again, the comparison between the two models demonstrates that the proposed Double-Weibull model is closer to simulation data.

\section{Discussion}

The analysis presented by Al-Habash et al. in [3] justified the use of the gamma-gamma distribution as an efficient tool for describing turbulence induced fading in FSO systems. A great advantage of this distribution is that its parameters can be estimated directly from atmospheric data. Our analysis showed that the proposed statistic model is more accurate than the gamma-gamma one for the cases of moderate and strong turbulence. More specifically, it yields a better fit with simulation data for both plane and spherical waves, especially at low probabilities, in most of the considered cases.

There are various reasons that justify the use of the DoubleWeibull pdf as a moderate-to-strong turbulence pdf model. First, it is more accurate than the widely used gamma-gamma pdf. Furthermore, its parameters are directly related with the atmospheric conditions. Finally, its pdf and cdf can be given in a closed form using the well-known Meijer-G function. Note, that Meijer's G-function is a standard built-in function in most of the well-known mathematical software packages such as MAPLE and MATHEMATICA. In addition, using [11, Eq. (18)], Meijer's G-functions can be written in terms of the more tractable generalized hypergeometric functions $[8$, Eq. (9.14.1)].

It has to be pointed out that the gamma-gamma distribution can be also used as an alternative efficient irradiance pdf, since it is given in a simpler closed form. Therefore the choice between gamma-gamma and Double-Weibull pdf depends on a tradeoff that has to be made between accuracy and calculation complexity.

\section{Performance Evaluation}

In this section, we study the performance of an FSO system which employs intensity-modulation/direct-detection with OOK, assuming that the laser beams propagate through a Double-Weibull turbulence channel, which is modeled as a multiplicative random process with Additive White Gaussian Noise (AWGN). The channel is considered as memoryless, stationary and ergodic, with independent and identically distributed intensity fading statistics. It is also assumed that both the transmitter and receiver have perfect channel state information (CSI) and that the irradiance remains constant over the symbol duration. The received optical signal can then be written as

$$
y=\eta I x+n,
$$

where $s=\eta I$ is the instantaneous intensity gain, $x$ represents the information bits and is either 0 or $1, n$ is the AWGN with zero mean and variance $\sigma_{n}^{2}=N_{o} / 2, \eta$ is the opticalto-electrical conversion coefficient and $I$ is the normalized irradiance. Based on the above assumptions, we can now evaluate the average BER and outage probability.

\section{A. Error Analysis}

The BER of IM/DD with OOK in the presence of AWGN is given by

$$
P_{e}=P(1) P(e \mid 1)+P(0) P(e \mid 0),
$$

where $P(1)$ and $P(0)$ are the probabilities of sending 1 and 0 bits, respectively, and $P(e \mid 1)$ and $P(e \mid 0)$ denote the conditional bit-error probabilities when the transmitted bit is 1 and 0 . Assuming that $P(1)=P(0)=0.5$ and $P(e \mid 1)=P(e \mid 0)$, the conditioned on $I$ error probabilities can be expressed as [12]

$$
P(e \mid I)=P(e \mid 1, I)=P(e \mid 0, I)=Q\left(\frac{\eta I}{\sqrt{2 N_{o}}}\right)
$$


where $Q(\cdot)$ is the Gaussian Q-function defined as $Q(x)=$ $\frac{1}{\sqrt{2 \pi}} \int_{x}^{\infty} \exp \left(-\frac{t^{2}}{2}\right) d t$ and related to the complementary error function $\operatorname{erfc}(\cdot)$ via $\operatorname{erfc}(x)=2 Q(\sqrt{2} x)$.

The average BER, $P_{b}(e)$, can be obtained by averaging (13) over the distribution of the fading coefficient, $I$, in (5), according to

$$
P_{e}=\int_{0}^{\infty} f_{I}(I)\left[\frac{1}{2} \operatorname{erfc}\left(\frac{\eta I}{2 \sqrt{N_{o}}}\right)\right] d I .
$$

The above integral can be evaluated in closed form by expressing the erfc $(\cdot)$ integrand via a Meijer's G-function using [13, Eq. (8.4.14.2)] and [13, Eq. (8.2.2.14)] and [11, Eq. (21)]. Thus, a closed-form solution is obtained as follows

$$
\begin{aligned}
P_{e} & =\frac{\beta_{2}(k l)^{\frac{1}{2}} k \xi^{2} \nu^{-1}}{2^{\frac{3}{2}}(2 \pi)^{\frac{l+k}{2}}-1+\frac{\nu}{2}+\frac{k+l}{2}(\xi-1)} \\
& \times G_{2 \nu, \xi(k+l)+\nu}^{\xi(k+l), 2 \nu}\left[\frac{\nu^{\nu} \xi^{-\xi \cdot(k+l)}}{\left(\Omega_{1} l\right)^{l \xi}\left(\Omega_{2} k\right)^{k \cdot \xi}}\right. \\
& \left.\times\left(\frac{\eta^{2}}{4 N_{o}}\right)^{-\nu} \mid \begin{array}{c}
\Delta(\nu ; 1), \frac{1}{2} \Delta(\nu ; 1) \\
I_{\xi}(l ; 1), I_{\xi}(k ; 1), \Delta(\nu ; 0)
\end{array}\right],
\end{aligned}
$$

where $I_{\xi}(j ; m) \triangleq \Delta\left(\xi ; \frac{j-1+m}{j}\right), \ldots, \Delta\left(\xi ; \frac{m}{j}\right)$ are integer numbers. Figure 5 depicts the BER for different degrees of turbulence severity that demonstrates the deterioration of the FSO system performance turbulence increases.

\section{B. Outage Probability}

The outage probability is defined as the probability that the instantaneous SNR falls below a specified threshold, $\gamma_{t h}$, which represents a protection value of the SNR above which the quality of the channel is satisfactory. The instantaneous electrical-to-noise ratio (SNR) can be defined as $\gamma=(\eta I)^{2} / N_{o}$, while the average electrical SNR is defined as $\mu=(\eta E\langle I\rangle)^{2} / N_{o}$. Since $E\langle I\rangle=1, I$ can be written as $I=\sqrt{\gamma / \mu}$. After the transformation of the random variable, $I$, the cfd of $\gamma$ is derived from (7) as

$$
\begin{aligned}
F_{\gamma}(\gamma) & =\frac{\sqrt{l k}}{(2 \pi)^{\frac{l+k}{2}-1}} \\
& \times G_{1, k+l+1}^{k+l, 1}\left[\frac{\left(\frac{\gamma}{\mu}\right)^{\frac{\beta_{1} \cdot l}{2}}}{\left(\Omega_{1} l\right)^{l}\left(\Omega_{2} k\right)^{k}} \mid \Delta(l ; 1), \Delta(k ; 1), 0\right] .
\end{aligned}
$$

Then, the outage probability is given as

$$
P_{\text {out }} \triangleq \operatorname{Pr}\left(\gamma \leq \gamma_{\text {th }}\right)=F_{\gamma}\left(\gamma_{\text {th }}\right) .
$$

The outage probability for moderate and strong turbulence is depicted in Fig. 6. The derived results show that the probability of outage increases as the turbulence strength gets stronger.

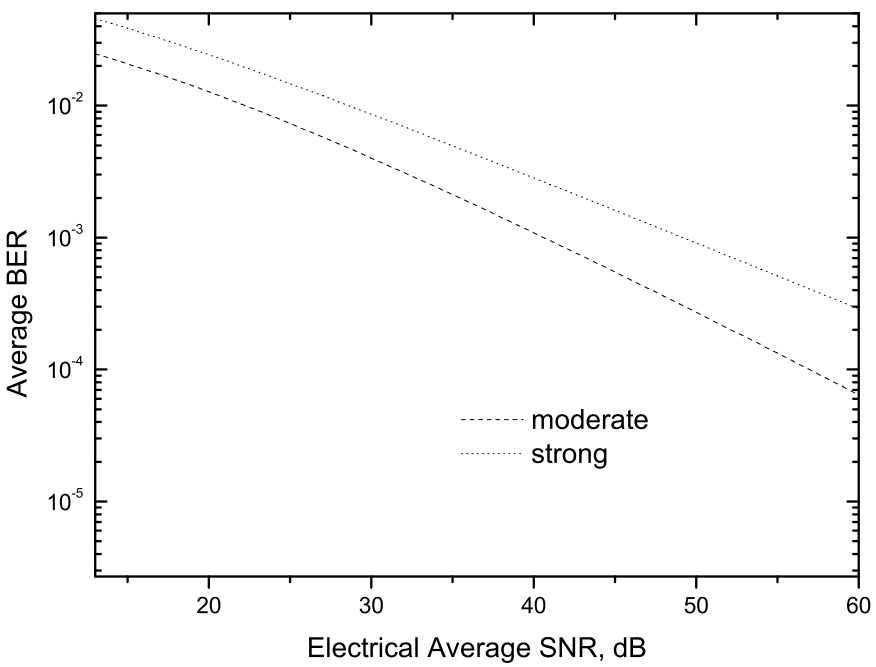

Fig. 5. Average BER as a function of $\mu$ for moderate and strong turbulence with Double-Weibull parameters as defined in Fig. 1.

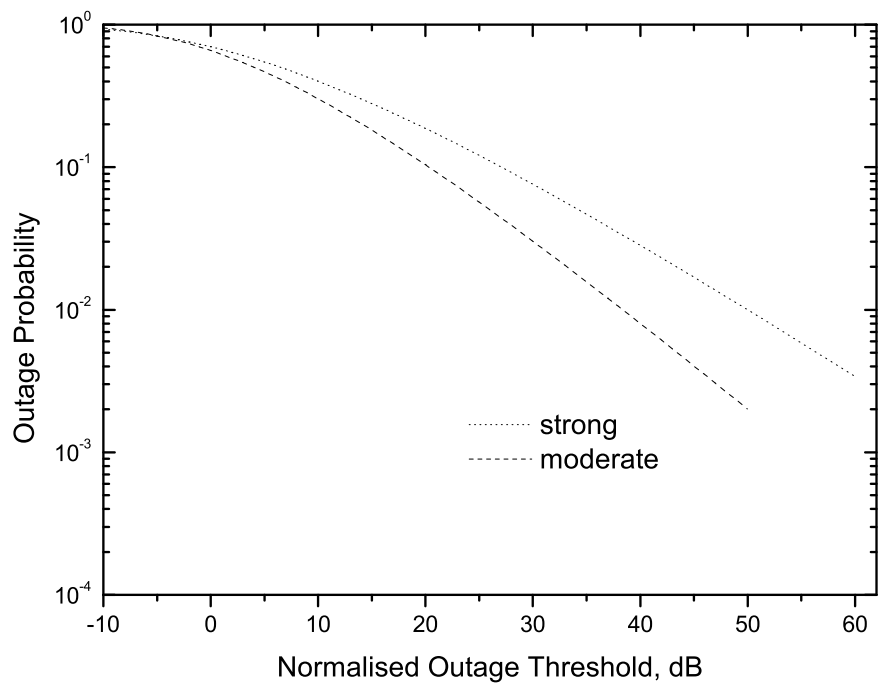

Fig. 6. Outage probability versus $\mu / \gamma_{t h}$ for moderate and strong turbulence with Double-Weibull parameters as defined in Figs. 1 and 2 respectively.

\section{CONCLUSiOns}

The identification of a tractable pdf to describe atmospheric turbulence is important in order to study the performance characteristics of an FSO system. In this paper, we have introduced a new channel model (Double-Weibull) which describes irradiance fluctuations in moderate and strong turbulence. We have obtained closed-form expressions for the pdf and cdf in terms of Meijer's G-function. Comparisons with the classical gamma-gamma pdf showed that the new model provides an accurate fit with numerical simulation data for both plane and spherical waves. Moreover, we have examined the BER of an IM/DD with OOK FSO system and the outage probability over the new turbulence model. Numerical results have shown the impact of turbulence severity on the overall performance. 


\section{REFERENCES}

[1] L. Andrews, R. L. Philips, and C. Y. Hopen, Laser Beam Scintillation with Applications. SPIE Press, 2001.

[2] A. Consortini, F. Cochetti, J. H. Churnside, and R. J. Hill, "Inner-scale effect on iradiance variance measured for weak-to-strong atmospheric scintillation," Journal of Optical Society of America A, vol. 10, pp. 2354 2362, 1993.

[3] M. A. Al-Habash, L. C. Andrews, and R. L. Philips, "Mathematical model for the irradiance probability density function of a laser beam propagating through turbulent media," Optical Engineering, vol. 40, pp. 1554-1562, Aug. 2001

[4] L. C. Andrews, R. L. Philips, and C. Y. Hopen, "Theory of optical scintillation," Journal of Optical Society of America A, vol. 16, no. 6, pp. 1417-1429, Jun. 1999.

[5] S. M. Flatté, C. Bracher, and G.-Y. Wang, "Probability-density functions of irradiance for waves in atmospheric turbulence calculated by numerical simulations," Journal of Optical Society of America A, vol. 11, no. 7, pp. 2080-2092, Jul. 1994

[6] R. J. Hill and R. G. Frehlich, "Probability distribution of irradiance for the onset of strong scintillation," Journal of Optical Society of America A, vol. 14, no. 7, pp. 1530-1540, Jul. 1997.

[7] K. Bury, Statistical Distributions in Engineering. Cambridge University Press, 1999.
[8] I. S. Gradshteyn and I. M. Ryzhik, Table of Integrals, Series, and Products, 7th ed. New York: Academic, 2007.

[9] N. C. Sagias, G. K. Karagiannidis, P. T. Mathiopoulos, and T. A. Tsiftsis, "On the performance analysis of equal-gain diversity receivers over generalised gamma fading channels," IEEE Trans. Wireless Commun., vol. 5, no. 10, pp. 2967-2975, Oct. 2006.

[10] K. Furutsu, "Theory of irradiance distribution function in turbulent media - cluster approximation," J. Math Phys., vol. 17, pp. 1252-1263, 1976.

[11] V. S. Adamchik and O. I. Marichev, "The algorithm for calculating integrals of hypergeometric type functions and its realization in REDUCE system," in Proc. International Conference on Symbolic and Algebraic Computation, Tokyo, Japan, 1990, pp. 212-224.

[12] T. A. Tsiftsis, H. G. Sandalidis, G. K. Karagiannidis, and M. Uysal, "Optical wireless links with spatial diversity over strong atmospheric turbulence channels," IEEE Trans. Wireless Commun., vol. 8, no. 2, pp. 951-957, Feb. 2009.

[13] A. P. Prudnikov, Y. A. Brychkov, and O. I. Marichev, Integrals and Series. More Special Functions. New York: Gordon and Breach Science Publishers, 1990, vol. 3, (first ed. in Moscow, Nauka, 1986). 